\title{
The bankruptcy problem: A cooperative bargaining approach
}

\author{
Nir Dagan* ${ }^{* \dagger}$ Oscar Volij \\ Department of Economics, Hebrew University of Jerusalem, Mount Scopus, 91905 Jerusalem, Israel \\ Communicated by M. Shubik \\ Received November 1992 \\ Revised April 1993
}

\begin{abstract}
We associate each bankruptcy problem with a bargaining problem and derive old allocation rules for the former by applying well known bargaining solutions to the latter.
\end{abstract}

Key words: Bankruptcy; Bargaining; Allocation

\section{Introduction}

Consider a situation in which a man dies and his estate is insufficient to meet his debts. The bankruptcy problem deals with the following question: how to divide the estate among all creditors. The natural approach to this problem would be to look for allocation rules that satisfy some desired properties. Two of the most well known and ancient rules are the proportional and the constrained equal award. The first one assigns awards in proportion to the claims while the second divides the estate equally among the creditors provided that no one gets more than his claim. O'Neill (1982), Aumann and Maschler (1985) and Curiel, Maschler and Tijs (1988) took an indirect approach. They used cooperative game theory as a tool to find some allocation rules and analyze their properties. They associated a TU game to each bankruptcy problem and applied to it well known single-valued solution concepts, which induce allocation rules for the bankruptcy problem. Solution concepts are usually required to be symmetric, Pareto efficient and invariant to strategic equivalence. Aumann and Maschler (1985) showed that if the bankruptcy problem is represented by a TU game, then it is impossible to obtain either the proportional or the constrained equal award rules out of a symmetric, Pareto efficient, and invariant to strategic equivalence solution concept applied to it. Accordingly, in order to get any of these rules we must either

\footnotetext{
* Corresponding author.

${ }^{+}$We wish to thank Nethanel Bar-Hacham and Sigmund Rehimai for useful comments.
} 
give up one of the three appealing properties, or change the game into an NTU one. We chose to try the second alternative. Although it seems very natural to treat the bankruptcy problem as a TU game since the question it poses is how to allocate a fixed amount of money, the notion of the worth of a coalition is not evident at all.

In this paper we adopt the indirect approach that defines allocation rules by means of solution concepts to pure bargaining problcms. Wc shall see that the Nash bargaining solution induces the constrained equal award rule, and that the Kalai-Smorodinsky bargaining solution induces the adjusted proportional rule introduced by Curiel, Maschler and Tijs (1988). Furthermore, some new rules are generated.

In the second section we define a bankruptcy problem as well as allocation rules. We give some examples and discuss some of their properties. We also give some definitions related to the bargaining problem. In the third section we define, for each bankruptcy problem, two different bargaining problems. We apply to them several bargaining solutions and analyze the corresponding allocation rules.

\section{Basic concepts and definitions}

A bankruptcy problem is a pair $(E, c)$ where $c=\left(c_{1}, \ldots, c_{n}\right) \geq \mathbf{0}$, and $0 \leq E \leq$ $\sum_{i=1}^{n} c_{i}$. E represents the total value of the estate and $c$ is the vector of the creditors' claims. The sum of these claims is denoted by $C$. An allocation in such a problem is an $n$-tuple $x=\left(x_{1}, \ldots, x_{n}\right) \in \mathbb{R}^{n}$ with $\sum_{i=1}^{n} x_{i}=E$ and $0 \leq x_{i} \leq c_{i}$, $i=1, \ldots, n{ }^{1}$ An allocation rule is a function that assigns a unique allocation to each bankruptcy problem. We denote the set of all creditors by $N$.

Examples:

(a) The proportional rule is defined as follows:

$$
g^{\mathrm{Pr}}(E, c)=\lambda c, \text { where } \lambda C=E .
$$

The proportional rule allocates awards proportionally to the claims. The proportionality principle was favored by the philosophers of ancient Greece and Aristotle even considered it as equivalent to justice. The proportional rule is widely used nowadays in many countries.

(b) The constrained equal award (CEA) rule is defined as follows:

$$
\begin{aligned}
g^{\mathrm{CEA}}(E, c)= & x \text { where } x_{i}=\min \left(\lambda, c_{i}\right) \text { and } \lambda \text { solves the equation } \\
& \sum_{i \in N} \min \left(\lambda, c_{i}\right)=E \mathrm{I}^{2}
\end{aligned}
$$

This rule assigns the same sum to all creditors as long as this sum does not exceed

\footnotetext{
${ }^{1}$ Note that for each well-defined bankruptcy problem, the set of its allocations is non-empty.

${ }^{2}$ This equation has a unique solution when $C>E$. If $C=E$, any solution $\lambda$ is greater than or equal to the maximum claim and therefore $x_{i}=c_{i}$ for all $i$.
} 
each creditor's claims. This rule is also very ancient, and was adopted by important rabbinical legislators, including Maimonides.

(c) Another rule, defined only for two-creditor problems, is the contested garment principle, which is defined as follows.

$$
g^{\mathrm{CG}}\left(E,\left(c_{1}, c_{2}\right)\right)=\left(\frac{E+c_{1}^{E}-c_{2}^{E}}{2}, \frac{E+c_{2}^{E}-c_{1}^{E}}{2}\right) .
$$

where $c_{i}^{E}=\min \left\{c_{i}, E\right\}$.

As a legal concept, $c_{i}^{E}$ may be interpreted as that part of $E$ claimed by creditor $i$. He cannot claim more than is there, thus $c_{i}^{E}$ can be interpreted as the relevant claim given $E$. The contested garment principle appears in the Babylonian Talmud (Baba Metzia, 2a) and its rationale will be explained below.

In order to define the next rule we need the following definition:

For each bankruptcy problem $(E, c)$ and creditor $i$, define

$$
v_{i}^{(E, c)}=\max \left\{0 ; E-\sum_{j \neq i} c_{j}\right\} .
$$

$v_{i}^{(E, c)}$ is the amount of money conceded to creditor $i$ by all other creditors. Whenever there is no danger of confusion we write simply $v_{i}$ instead of $v_{i}^{(E, c)}$. We will show that if $\boldsymbol{x}$ is an allocation, then $v_{i} \leq x_{i}$, which mcans that $v_{i}$ is the minimum amount of money that may be assigned to $i$ by any allocation rule.

(d) The adjusted proportional $(A P)$ rule is defined as follows: $g^{\mathrm{AP}}(E, c)=\boldsymbol{x}$, where

$$
x_{i}= \begin{cases}v_{i}+\left(c_{i}^{E}-v_{i}\right)\left(\sum_{j \in N}\left(c_{j}^{E}-v_{j}\right)\right)^{-1}\left(E-\sum_{j \in N} v_{j}\right) & \text { if } C>E>0 \\ c_{i} & \text { if } E=C \\ 0 & \text { if } E=0\end{cases}
$$

This rule is a generalization of the contested garment principle for $n$-creditor bankruptcy problems, and was introduced by Curiel, Maschler and Tijs (1988). ${ }^{3}$ $g^{\mathrm{AP}}$ can be interpreted as allocating $E$ in two stages. In the first stage, each creditor $i$ gets whatever the others concede, that is, he gets $v_{i}$, leaving $E-\Sigma_{i \in N} v_{i}$ for the second stage. In this last stage $g^{\mathrm{AP}}$ divides the remainder in proportion to the outstanding relevant claims. The same interpretation applies to the contested garment principle, since it is a particular case of $g^{\text {AP }}$.

Some properties may be required of allocation rules. We shall discuss some them.

(i) Independence of irrelevant claims. A rule $g$ is said to satisfy independence of irrelevant claims if for each bankruptcy problem $(E, c), g(E, c)=g\left(E, c^{E}\right)$, where $c^{E}=\left(c_{1}^{E}, \ldots, c_{n}^{E}\right)$.

A rule $g$ is independent of irrelevant claims, if it allocates the estate taking into

\footnotetext{
${ }^{3}$ Their formulation of the rule is slightly different, but it is shown in Lemma 2 in Section 3 that it is equivalent to ours.
} 
account only the relevant claims. $g^{\mathrm{CEA}}$ and $g^{\mathrm{AP}}$ both satisfy independence of irrelevant claims. $g^{\mathrm{Pr}}$ does not.

The way $v_{i}$ is defined, together with the fact that any allocation assigns each player an amount greater than $v_{i}$, allows us to interpret $v_{i}$ as the maximum amount of money about which everybody agrees is due to creditor $i$. No matter how the estate is divided among the creditors, there is complete agreement that each will receive at least $v_{i}$. This inspires the following property:

(ii) $v$-Separability. An allocation rule $g$ is $v$-separable if for each bankruptcy problem $(E, c)$,

$$
g(E, c)=\boldsymbol{v}+g\left(E-\sum_{i \in N} v_{i}, c-v\right)
$$

where $\boldsymbol{v}=\left(v_{1}, \ldots, v_{n}\right)$.

$v$-separability says that the problem can be solved in two steps: first give each creditor the amount conceded by the others and then apply the rule to the bankruptcy problem where the debts are reduced by $v$ and the value of the assets is what remains after the first step.

It can be shown that $\left(E-\sum_{i \in N} v_{i}, c-v\right)$ is indeed a bankruptcy problem. To see this, let $\boldsymbol{x}$ be an allocation of the original problem $(E, c)$. Clearly, by definition of allocation, $x_{i} \leq c_{i}$ for all $i$. If $v_{i}=0$ then $v_{i} \leq x_{i} \leq c_{i}$. If $v_{i}=$ $E-\sum_{j \neq i} c_{j}$ then $v_{i}=E-\Sigma_{j \neq i} c_{j} \leq E-\Sigma_{j \neq i} x_{j}=x_{i}$. Hence $v_{i} \leq x_{i} \leq c_{i}$ and therefore $\boldsymbol{c}-\boldsymbol{v} \geq \mathbf{0}$. Furthermore, $E-\Sigma_{i \in N} v_{i} \geq E-\Sigma_{i \subset N} x_{i}=0$.

$v$-Separability is not satisfied by $g^{\mathrm{Pr}}$ nor by $g^{\mathrm{CEA}}$. It is satisfied by $g^{\mathrm{AP}}$.

(iii) Zero out. Let $(E, \mathbf{c})$ be a bankruptcy problem. A creditor $i$ is called a zero if $c_{i}=0$. Denote by $Q$ the set of all non-zero creditors. A rule $g$ satisfies zero out if $g(E, c)=x \Rightarrow g(E, c \mid Q)=x \mid Q$. If a rule satisfies zero out, then the amount assigned to the non-zero creditors cannot be 'manipulated' by subtracting or adding zero creditors. The proportional, CEA and the AP rules satisfy zero out.

We would like to see the solution to a given bankruptcy problem as a result of a bargaining process among the creditors. Before we do this we need some definitions.

A bargaining problem is a pair $(S, \boldsymbol{d})$ where $S \subset \mathbb{R}^{n}$ is a compact and convex set, $\boldsymbol{d} \in S$ and there exist $s \in S$ such that $s_{i}>d_{i}, i=1, \ldots, n .(S, d)$ represents a situation where $n$ individuals bargain over a set of possible agreements. $S$ is the set of all feasible utility allocations that may be reached by means of an agreement. If there is no agreement, each individual $i$ gets the level of utility $d_{i}$. We denote the set of individuals participating in the bargaining process by $N$.

A bargaining solution is a function $f$ that assigns cach bargaining problcm $(S, d)$ a unique element of $S$.

Examples of bargaining solutions:

(a) The Nash bargaining solution (Nash, 1950) is defined in the following way:

$$
f^{N}(S, \boldsymbol{d})=\underset{d \leq x \in S}{\operatorname{argmax}} \prod_{i=1}^{n}\left(x_{i}-d_{i}\right) .
$$


(b) Let $\Delta=\left\{\alpha \in \mathbb{R}^{n} \mid \alpha_{i}>0\right\}$. The $\alpha$-asymmetric Nash solution as:

$$
f^{\alpha}(s, d)=\underset{d \leq x \in S}{\operatorname{argmax}} \prod_{i=1}^{n}\left(x_{i}-d_{i}\right)^{\alpha_{i}} .
$$

The family $\left\{f^{\alpha}\right\}_{\alpha \in \Delta}$ is known as the family of asymmetric Nash solutions.

(c) The Kalai-Smorodinsky solution (Kalai and Smorodinsky, 1975): For each bargaining problem $(S, \boldsymbol{d})$ let $\operatorname{IR}(S, \boldsymbol{d})=\{\boldsymbol{x} \subset S \mid \boldsymbol{x} \geq \boldsymbol{d}\}$ and $\boldsymbol{a}_{\boldsymbol{i}}(S, \boldsymbol{d})=$ $\max \left\{x_{i} \mid\left(x_{i}, x_{-i}\right) \in \operatorname{IR}(S, \boldsymbol{d})\right\}, \quad i=1, \ldots, n$. The ideal point $\boldsymbol{a}(S, \boldsymbol{d}) \equiv$ $\left(a_{1}(S, d), \ldots, a_{n}(S, d)\right)$ gives the maximum obtainable utility levels of each agent, subject to the condition that all agents achieve at least the utility levels of the disagreement point. The function $f^{\mathrm{KS}}$ assigns to each bargaining problem $(S, d)$ the unique weak Pareto optimal member of $S$ on the line joining $d$ and $a(S, d)$.

Some properties are often required of bargaining solutions. Some of these are:

(i) Pareto optimality. Suppose $(S, \boldsymbol{d})$ is a bargaining problem and $y \in S . f$ is said to satisfy Pareto optimality if $\boldsymbol{y} \geq f(S, \boldsymbol{d})$ implies $\boldsymbol{y}=f(S, \boldsymbol{d})$.

(ii) Translation invariance. For all bargaining problems $(S, \boldsymbol{d})$ and for all $\boldsymbol{t} \in \mathbb{R}^{n}, f(S+\{\boldsymbol{t}\}, \boldsymbol{d}+\boldsymbol{t})=f(S, \boldsymbol{d})+\boldsymbol{t}$.

(iii) Independence of non-individually-rational alternatives (INIR). When we defincd the Kalai-Smorodinsky bargaining solution we also defined, for any bargaining problem $(S, d)$ the set $\operatorname{IR}(S, d)$. It is sometimes natural to ask that a bargaining solution be independent of alternatives that lie outside the set $\operatorname{IR}(S, d)$. When this is the case, we say that the bargaining solution satisfies independence of non-individually-rational alternatives. Formally: a bargaining solution satisfies INIR if for each bargaining problem $(S, d), f(S, d)=f(\operatorname{IR}(S, d)$, d).

(iv) Independence of irrelevant alternatives $(I I A)$. Let $\left(S_{1}, d\right)$ and $\left(S_{2}, d\right)$ be two bargaining problems with $S_{1} \subset S_{2}$. $f$ satisfies IIA if, whenever $f\left(S_{2}, d\right) \in S_{1}$, it is also true that $f\left(S_{2}, \boldsymbol{d}\right)=f\left(S_{1}, \boldsymbol{d}\right)$.

It is well known that the family of asymmetric Nash solutions satisfy Pareto optimality, translation invariance and IIA. It also satisfies INIR. The KalaiSmorodinsky solution satisfies translation invariance and INIR but not IIA nor Parcto optimality.

\section{Bankruptcy as a bargaining problem}

We now define a bargaining problem associated with each bankruptcy problem. For this purpose it is necessary to define a compact convex set $S$ and a disagreement point $d \in S$ in terms of each bankruptcy problem $(E, c)$. We propose that the appropriate set $S$ be defined as:

$$
S(E, c)=\left\{x \in \mathbb{R}_{+}^{n} \mid x \leq c, \sum_{i \in N} x_{i} \leq E\right\} .
$$


This means that the individuals are bargaining over all the possible divisions of the estate $E$ that give each one no more than his claim. In order to complete the definition of the bargaining problem we must set the disagreement point $\boldsymbol{d}$. But before we discuss the choice of $\boldsymbol{d}$, notice that no matter how $\boldsymbol{d}$ is chosen, if we want a bargaining solution to assign to every associated bargaining problem $(S(E, c), \boldsymbol{d})$ an allocation to the bankruptcy problem $(E, c)$, we must require that the bargaining solution should satisfy Pareto optimality on the class of bargaining problems that arise from bankruptcy problems. ${ }^{4}$ The choice of the disagreement point is not as natural as was the choice of the set of feasible agreements. We consider two alternatives that seem to be the more natural ones. The first alternative is to define the disagreement point as $\mathbf{0}$. This means that if the creditors fail to reach an agreement, no one gets anything. In order to get something, they need to reach a unanimous agreement.

We now define for each bankruptcy problem $(E, c)$ the $\mathbf{0}$-associated bargaining problem as $(S(E, c), 0) .^{5}$ As we noted before, each bargaining solution that satisfies Pareto optimality is an allocation rule for the bankruptcy problem when applied to the associated bargaining problem. We want to know which rules are induced by different bargaining solutions.

Proposition 1. The Nash bargaining solution induces the constrained equal award rule, i.e. $f^{N}(S(E, c), \mathbf{0})=g^{\mathrm{CEA}}(E, \boldsymbol{c})$.

Proof. Let $(E, c)$ be a bankruptcy problem with $\boldsymbol{c} \gg \mathbf{0}$ and $E>0$. The allocation assigned by $f^{N}$ to the $\mathbf{0}$-associated problem $(S(E, c), \mathbf{0})$ is the unique solution to the following constrained maximization problem:

$$
\begin{aligned}
& \max \prod_{i=1}^{n} x_{i} \\
& \text { s.t. } 0 \leq x_{i} \leq c_{i} \forall i \in N \\
& \sum_{i=1}^{n} x_{i} \leq E .
\end{aligned}
$$

Let $\boldsymbol{x}^{*}$ be this solution. Since the Nash solution satisfies Pareto optimality, we know that $\sum_{i \in N} x_{i}^{*}=E$. There are two cases. In the first case $x_{i}^{*}=c_{i}$ for all $i \in N$. In this case, by Pareto optimality we have $C=E$ and therefore the allocation $x^{*}$ coincides with the constrained equal award allocation. In the second case, $x_{i}^{*}<c_{i}$ for some $i \in N$. Let $i$ be a creditor for whom, $x_{i}^{*}<c_{i}$ and denote $x_{i}^{*}$ by $\lambda$. It must

\footnotetext{
${ }^{4}$ Although the Kalai-Smorodinsky bargaining solution does not satisfy Pareto optimality in general, it does so on this class.

${ }^{5}$ This hargaining problem is well defined if and only if there are no zero creditors and $E>0$. We restrict ourselves to bankruptcy problems that satisfy these conditions. On the other hand, rules induced by bargaining solutions can be uniquely extended to all bankruptcy problems by zero out. The extended rule is the unique rule which coincides with the induced rule wherever it is defined and satisfies zero out. It can be verified that all the propositions below hold for the extended rules as well.
} 
be the case that

$$
x_{j}^{*} \leq \lambda \text { for all } j \in N
$$

for otherwise we could transfer a small amount from $j$ to $i$ while still satisfying the constraints in (1) and thus bringing the maximand to a higher value. On the other hand, the constraints in (1) require that $x_{j}^{*} \leq c_{j}$ for all $j$ which, together with (2), implies that $x_{j}^{*} \leq \min \left\{\lambda, c_{j}\right\}$. But this inequality cannot be strict because, by an argument analogous to the one that justified (2), if $x_{j}^{*}<c_{j}$ then $x_{j}^{*}=\lambda$.

We can use the asymmetric Nash solution to reflect the asymmetry in the creditors' claims.

Proposition 2. The $\alpha$-asymmetric Nash bargaining solution for $\alpha=c$ induces the proportional rule, i.e. $f^{c}(S(E, c), \mathbf{0})=g^{\mathrm{Pr}}(E, c)$.

Proof. Let $(E, c)$ be a bankruptcy problem with $\boldsymbol{c} \gg \boldsymbol{0}$ and $E>0$. Consider the following bargaining problem $(\boldsymbol{F}, \mathbf{0})$, where $F=\left\{\boldsymbol{x} \in \mathbb{R}_{+}^{n} \mid \boldsymbol{x} \geq \mathbf{0}, \Sigma_{i \in N} x_{i} \leq E\right\}$. Let $f^{c}(F, \mathbf{0})=\boldsymbol{x}^{*}$. From the first order conditions of the maximization problem that defines the asymmetric Nash solution it follows that:

$$
\frac{x_{i}^{*}}{x_{j}^{*}}=\frac{c_{i}}{c_{j}} \forall i, j \in N .
$$

Furthermore, $\Sigma_{i \in N} x_{i}^{*}=E$ by Pareto optimality, which implies $f^{c}(F, \mathbf{0})=\boldsymbol{x}^{*}=$ $g^{\operatorname{Pr}}(E, c)$. Hence $x^{*} \in S(E, c)$. On the other hand, $S(E, c) \subset F$. Therefore, by IIA, $f^{c}(S(E, c), \mathbf{0})=f^{c}(F, \mathbf{0})=\boldsymbol{x}^{*}=g^{\mathrm{Pr}}(E, c)$.

Application of the Kalai-Smorodinsky bargaining solution yields a new rule which is, in a sense, halfway between the proportional and the AP rules.

Proposition 3. The Kalai-Smorodinsky bargaining solution applied to the $\mathbf{0}$ associated bargaining problem allocates the estate in proportion to the relevant claims. Formally, for each bankruptcy problem $(E, c), f^{\mathrm{KS}}(S(E, c), \quad \mathbf{0})=$ $g^{\mathrm{Pr}}\left(E, c^{E}\right)$.

Proof. Let $(E, c)$ be a bankruptcy problem with $c \gg 0$ and $E>0$ and let $S(E, c), \mathbf{0})$ be the corresponding $\mathbf{0}$-associated bargaining problem. It is easy to verify that the ideal point $\boldsymbol{a}(S(E, c), \mathbf{0})$ equals the relevant claims $c^{E}$. Denote $g^{\operatorname{Pr}}\left(E, c^{E}\right)$ by $\boldsymbol{x}^{*}$. That is $\boldsymbol{x}^{*}=t c^{E}, \Sigma_{i \in N} x_{i}^{*}=E$. But then $\boldsymbol{x}^{*}$ is an efficient point on the line segment that joins $a(S(E, c), 0)$ with 0 . Hence it must be $f^{\mathrm{KS}}(S(E, c), \mathbf{0})$.

As a straightforward corollary of the previous proposition we have that the rule defined by the Kalai-Smorodinsky bargaining solution, when applied to the $\mathbf{0}$-associated bargaining problem, satisfies independence of irrelevant claims. On 
the other hand, it is easily verified that this rule does not satisfy $v$-separability. This rule is interesting as it tries to reconcile the proportionality principle with the independence of irrelevant claims property. It treats each dollar of relevant claims equally, and ignores any dollar claimed in excess of the total estate. While this modified proportional rule has no importance of its own, it will help us proving Proposition 4 and understanding the AP rule.

Now we turn to the second alternative for the choice of the disagrecment point. According to this approach, we define for each bankruptcy problem $(E, c)$ the $\boldsymbol{v}$-associated bargaining problem as $\left(S(E, c), \boldsymbol{v}^{(E, c)}\right)$ (see definition of $\left.\boldsymbol{v}\right){ }^{6}$ The more interesting result under this alternative concerns the Kalai-Smorodinsky bargaining solution.

Proposition 4. The Kalai-Smorodinsky bargaining solution applied to the $\boldsymbol{v}$ associated bargaining problem induces the $A P$ rule, i.e. $f^{\mathrm{KS}}(S(E, c), v)=$ $g^{\mathrm{AP}}(E, c)$.

To show this we need the following two lemmas:

Lemma 1. Let $f$ be a bargaining solution that satisfies translation invariance and INIR. Then, the allocation rule defined by $f$ when applied to the $v$-associated bargaining problem is v-separable, i.e. for each bankruptcy problem $(E, c)$, $f(S(E, \boldsymbol{c}), \boldsymbol{v})=\boldsymbol{v}+f\left(S\left(E-\Sigma_{i \in N} v_{i}, \boldsymbol{c}-\boldsymbol{v}\right), \mathbf{0}\right)$.

Proof. Since $f$ satisfies translation invariance, $f(S(E, c), \boldsymbol{v})=\boldsymbol{v}+f(S(E, \boldsymbol{c})-\{\boldsymbol{v}\}$, 0). By INIR, $f(S(F, c)-\{\boldsymbol{v}\}, \mathbf{0})=f(\operatorname{IR}(S(E, c)-\{\boldsymbol{v}\}, \mathbf{0}), \mathbf{0})$. But it is easily seen that $\operatorname{IR}(S(E, \boldsymbol{c})-\{\boldsymbol{v}\}, \mathbf{0})=S\left(E-\sum_{i \in N} v_{i}, \boldsymbol{c}-\boldsymbol{v}\right)$, therefore $f(S(E, \boldsymbol{c}), \boldsymbol{v})=\boldsymbol{v}+$ $f\left(S\left(E-\Sigma_{i \in N} v_{i}, \boldsymbol{c}-\boldsymbol{v}\right), \mathbf{0}\right)$.

Since both the Nash and the Kalai-Smorodinsky bargaining solutions satisfy INIR and translation invariance, we have the following:

\section{Corollary.}

$$
\begin{aligned}
& f^{\mathrm{KS}}(S(E, \boldsymbol{c}), \boldsymbol{v})=\boldsymbol{v}+g^{\operatorname{Pr}}\left(E-\sum_{i \in N} v_{i},(\boldsymbol{c}-\boldsymbol{v})^{E-\Sigma_{i \in N} v_{i}}\right) \\
& \left.f^{\mathrm{N}}(S(E, \boldsymbol{c}), \boldsymbol{v})\right)=\boldsymbol{v}+g^{\mathrm{CEA}}\left(E-\sum_{i \in N} v_{i}, \boldsymbol{c}-\boldsymbol{v}\right) .
\end{aligned}
$$

Proof. Apply Lemma 1 together with Propositions 3 and 1 in order to prove (1) and (2) respectively.

\footnotetext{
${ }^{6}$ Again, the $\boldsymbol{v}$-associated bargaining problem is well defined if and only if $E>0$, there are no zero creditors, and $C>E$. As before, we restrict ourselves to these cases although all the propositions hold for the extended rules as well (see footnote 5).
} 
It is clear from the corollary that rules (1) and (2) satisfy independence of irrelevant claims. It can also be verified that these rules coincide with the contested garment principle for two-creditor bankruptcy problems. (For more on this coincidence, see Dagan, 1992).

Lemma 2. For each bankruptcy problem $(E, c)$ and for all $i \in N$, the following identity is satisfied: $\left(c_{i}-v_{i}\right)^{E-\Sigma_{i \in N} v_{i}}=c_{i}^{E}-v_{i}$.

Proof. See Appendix.

Proof of Proposition 4. It follows from the corollary of Lemma 1 and Lemma 2 that $f^{\mathrm{KS}}(S(E, \boldsymbol{c}), \boldsymbol{v})=\boldsymbol{v}+g^{\mathrm{Pr}}\left(E-\Sigma_{i \in N} v_{i}, c^{E}-\boldsymbol{v}\right)$ which by definition of the AP rule is identical to $g^{\mathrm{AP}}(E, c)$.

The AP rule tries to reconcile the principles of proportionality, independence of irrelevant claims and $v$-separability. To see this recall that the rule allocates the estate in two stages. In the first stage it gives the creditors their corresponding $v_{i}$ 's. In the second stage it divides the remainder in proportion to the outstanding relevant claims.

\section{Concluding comments}

O'Neill (1982), Aumann and Maschler (1985) and Curiel, Maschler and Tijs (1988) applied a game theoretic model in order to produce allocation rules for the bankruptcy problem. None of them derived either the CEA or the proportional rule for their model. In this paper we applied a similar approach, but used a pure bargaining model instead of a TU game. This enabled us to derive the CEA rule using a game theoretic solution concept, which is symmetric, translation invariant, and Pareto optimal. This result cannot be obtained using the TU model. Moreover, although our approach does not take into account coalitional aspects of the bankruptcy problem, we managed to derive the AP rule introduced by Curiel, Maschler and Tijs (1988).

We did not apply the Maschler-Perles (1981) bargaining solution to our model since, as far as we know, it is not generalized to $n$-person bargaining problems. However, it can easily be verified that, when applied to the $\mathbf{0}$-associated bargaining problem, the Maschler-Perles bargaining solution induces the contested garment principle. This result is quite surprising, since we could not get a $v$-separable rule from application of the other bargaining solutions to the $\mathbf{0}$ associated problem. It would be very interesting to check what allocation rule is induced by the generalization of the Maschler-Perles bargaining solution to $n$-person bargaining problems, when and if such generalization appears.

One property of allocation rules with which we did not deal, is consistency. Suppose a rule assigns some allocation to a given bankruptcy problem. Suppose, further, that a subset of creditors wants to allocate the total amount assigned to 
them applying the same rule. Consistency requires that the new allocation should be identical to the original onc. This property was introduced by Aumann and Maschler (1985) in the bankruptcy context. The analogue of consistency in the context of bargaining is called stability, and was useful in the characterizations of the Nash bargaining solution due to Harsanyi (1959) and Lensberg (1988). It can be shown that stable and Pareto optimal bargaining solutions induce consistent bankruptcy allocation rules, when applied to the $\mathbf{0}$-associated bargaining problems.

Allocation rules may-and in fact most of them do-satisfy various monotonicity properties. One of them, estate monotonicity, requires that when the estate increases, no creditor loses. Although desired, these monotonicity properties, are not restrictive since all interesting allocation rules satisfy them. This is in contrast to monotonicity properties in TU games and in bargaining problems, where they proved to be powerful for characterization results (see, for example, Young, 1985; Kalai and Smorodinsky, 1975; Thomson, 1983, 1985).

Another way to better understand different properties of allocation rules would be to apply a non-cooperative approach to the bankruptcy problem. The idea is to derive allocation rules from equilibrium payoffs of a corresponding non-cooperative game. This idea is due to O'Neill (1982). Recent advances in this direction are due to Serrano (1992) who, based on consistency and monotonicity, succeeded in implementing the contested-garment-consistent allocation rule (see Aumann and Maschler, 1985). We believe that other consistent allocation rules can be implemented by similar procedures. This is a subject for further research.

\section{Appendix}

Lemma 2. For each bankruptcy problem $(E, c)$ and for all $i \in N$, the following is satisfied: $\left(c_{i}-v_{i}\right)^{E-\Sigma_{i \in N} v_{i}}=c_{i}^{E}-v_{i}$.

Proof. Let $(E, c)$ be a bankruptcy problem. For cach creditor $i$ define the following set: $A(i)=\left\{j \in N \backslash\{i\} \mid v_{j}>0\right\}$. Take a creditor $j$. There are two cases. Case 1: $A(j)=\emptyset$.

In this case, $\Sigma_{i \in N} v_{i}=v_{j}$, hence $\left(c_{j}-v_{j}\right)^{E-\Sigma_{i \in N} v_{i}}=\left(c_{j}-v_{j}\right)^{E-v_{j}}=c_{j}^{E}-v_{j}$. Case 2: $A(j) \neq \emptyset$.

In this case, $\exists j^{*} \neq j$ such that $v_{j^{*}}>0$.

$$
\begin{aligned}
\sum_{i \in N \backslash\{j\}} v_{i} & =v_{j^{*}}+\sum_{i \in A(j) \cap A\left(j^{*}\right)}\left(E-\sum_{k \in N \backslash\{i\}} c_{k}\right) \\
& =E-\sum_{i \in A(j) \cap A\left(j^{*}\right)} c_{i}-\sum_{i \notin \in A(j)} c_{i}+\sum_{i \in A(j) \cap A\left(j^{*}\right)}\left(E-\sum_{k \in N \backslash\{i\}} c_{k}\right) \\
& =E+\sum_{i \in A(j) \cap A\left(j^{*}\right)}\left(E-\sum_{k \in N} c_{k}\right)-\sum_{i \notin \in A(j)} c_{i} \\
& \leq E-\sum_{i \notin A(j)} c_{i} \leq E-c_{j} .
\end{aligned}
$$


Hence, $c_{j}-v_{j} \leq E-\sum_{i \in N} v_{i}$, which implies $\left(c_{j}-v_{j}\right)^{E-\Sigma_{i \in N} v_{i}}=\left(c_{j}-v_{j}\right)$. And since $v_{j^{*}}>0, c_{j}<E$ and $c_{j}=c_{j}^{E}$, the result follows.

\section{References}

R.J. Aumann and M. Maschler, Game theoretic analysis of a bankruptcy problem from the Talmud,

J. Econom. Theory 36 (1985) 195-213.

I.J. Curiel, M. Maschler and S.H. Tijs, Bankruptcy games, Z. Op. Res 31 (1988) A143-A159.

N. Dagan, Characterizations of rules of the bankruptcy problem, Mimeo, 1992.

J.C. Harsanyi, A bargaining model for the cooperative $n$-person game, in: A.W. Tucker and R.D. Luce, eds., Contributions to the Theory of Games IV, Annals of Mathematics Studies 40 (Princeton Iniversity Press, Princeton, N.J, 1959).

E. Kalai and M. Smorodinsky, Other solution to the Nash's bargaining problem, Econometrica 43 (1975) 513-518.

T. Lensberg, Stability and the Nash solution, J. Econom. Theory 45 (1988) $330-341$.

M. Maschler and M. Perles, The super-additive solution for the Nash bargaining game, Int. J. Game Theory 10 (1981) 163-193.

J. Nash, The bargaining problem, Econometrica 18 (1950) 155-162.

B. O'Neill, A problem of rights arbitration from the Talmud, Math. Soc. Sci. 2 (1982) 345-371.

R. Serrano, Strategic bargaining, bankruptcy problems, and the Nucleolus, Mimeo, 1992.

W. Thomson, The fair division of a fixed supply among a growing population, Math. Oper. Res. 8 (1983) 319-326.

W. Thomson, Axiomatic theory of bargaining with a variable population: A survey of recent results, in: A.E. Roth, ed., Game Theoretic Models of Bargaining (Cambridge University Press, Cambridge, MA, 1985) pp. 233-258.

H.P. Young, Monotonic solutions of cooperative games. Int. J. Game Theory 14 (1985) 65-72. 